\title{
SCHUTZ VOR DISKRIMINIERUNGEN IN DEM BEREICH DER BESCHÄFTIGUNG IN DEM POLNISCHEN UND IN DEM DEUTSCHEN RECHT IM LICHT DER EUROPÄISCHEN RICHTLINIEN
}

\author{
JUSTYNA NOWAKOWSKA-GANCARZ*
}

\section{EINFÜHRUNG}

Die Gleichheit aller Menschen vor dem Gesetz und der Schutz vor Diskriminierung ist ein allgemeines Menschenrecht. Der Begriff Diskriminierung (lat. diskriminare) bedeutet trennen, unterscheiden. ${ }^{1}$ Darunter versteht man ungerechtfertigte Ungleichbehandlung, Benachteiligung, bzw. Herabwürdigung aufgrund bestimmter innerer oder äußerer Merkmale. Ungleiche Behandlungen, die im Zusammenhang mit der Rasse, der ethnischen Herkunft, Religion, Weltanschauung, Alter, Behinderung, sexuellen Orientierung stehen, sind in Europa ein verbreitetes Phänomen. Diese Erscheinung verstößt gegen die Grundrechte, die vor allem durch das Rechtssystem der Europäischen Union sowie durch völkerrechtliche Verträge unterstützt werden. Die Europäische Union dient nicht nur Wirtschaftszwecken - sie realisiert die Aufgabe der "Verteidigung der Grundrechte", weil die ungleiche Behandlung der Menschen gegen die Grundsätze der Freiheit, der Demokratie, der Achtung, der Menschenrechte und Grundfreiheiten verstößt. Das Problem der Diskriminierungen betrifft alle Bereiche des Lebens. Die Arbeitswelt ist der Bereich, der besonders diskriminierungsanfällig ist. Diskriminierungen am Arbeitsplatz sind ein Störfaktor im gemeinsamen Markt, sie können die Erreichung eines hohen Beschäftigungsniveaus und eines hohen Maßes an sozialem Schutz verhindern und hemmen so die die Steigerung von Lebensstandard und Lebensqualität. $^{2}$

DOI: $10.2478 /$ wrlae-2013-0029

* Magister; Doktorandin; Wroclaw Universität, Lehrstuhl für Völkerrecht und Europarecht; j.nowakowskagancarz@gmail.com

${ }^{1}$ Hans Schultz, Otto Basler, Deutsches Fremdwörterbuch (Institut für Deutsche Sprache 1999) 666.

2 Die Richtlinie 2000/43/EG vom 29. Juni 2000 zur Anwendung des Gleichbehandlungsgrundsatzes ohne Unterschied der Rasse oder der ethnischen Herkunft [2007] OJ L180/22. 
Mit vorliegendem Artikel sollen daher die arbeitsrechtlichen Regelungen zum Diskriminierungsschutz unter besonderer Berücksichtigung des polnischen und deutschen Rechts analysiert werden. Zunächst werden die maßgebenden europarechtlichen Vorschriften dargestellt, die die Basis für entsprechende nationale Gesetze geschaffen haben. Die zentralen Gleichbehandlungsrichtlinien verpflichten die Mitgliedsstaaten im Hinblick auf Diskriminierungen zur Gewährleistung bestimmter Mindeststandards hinsichtlich des Rechtsschutzes. Nachfolgend werden die innenstaatlichen Regelungen betrachtet, die Polen und Deutschland eingeführt haben, um den Verpflichtungen aus den genannten Richtlinien nachzukommen.

\section{SCHUTZ DER MENSCHENRECHTE AUF VÖLKERRECHTLICHER UND EUROPÄISCHER EBENE}

Im Prozess der europäischen Integration werden die Grundrechte auf verschiedenen Ebenen seit langem geschützt. Bereits die Art. 2 und 7 der allgemeinen Menschenrechtserklärung aus dem Jahre 1948 enthalten ein allgemeines Diskriminierungsverbot und betonen sowohl die Gleichheit aller Menschen vor dem Gesetz und den gleichen Schutz durch das Gesetz. ${ }^{3}$

Von großer Bedeutung ist die Konvention zum Schutze der Menschenrechte und Grundfreiheiten vom 4.11.1950, deren Vertragsstaaten alle 46 Mitgliedsstaaten des Europarates sind. ${ }^{4}$ Zusammen mit den Zusatzprotokollen enthält die Konvention eine große Anzahl von Rechten, wie beispielsweise das Recht auf Leben, das Verbot von Folter, das Verbot der Sklaverei, das Recht auf Freiheit und Sicherheit, das Recht auf Achtung des Privat- und Familienlebens, Gedanken-, Gewissens-, Religionsfreiheit. ${ }^{5}$ Laut Art. 14 der Konvention muss der Genuss, der in der Konvention festgelegten Rechte "ohne Unterschied des Geschlechts, der Rasse, Hautfarbe, Sprache, Religion, politischen oder sonstigen Anschauungen, nationaler oder sozialer Herkunft, Zugehörigkeit zu einer nationalen Minderheit, des Vermögens, der Geburt oder des sonstigen Status" gesichert werden. ${ }^{6}$ Daneben wurden Gleichheit vor dem Gesetz und Schutz vor Diskriminierungen auch in weiteren völkerrechtlichen Menschenrechtsverträgen gewährleistet. Zahlreiche Instrumente, die im Rahmen der Vereinten Nationen verabschiedet wurden, beinhalten Vorschriften zur Bekämpfung von Diskriminierungen. Darunter sind Der Internationale Pakt über bürgerliche und Politische Rechte vom 19.12.1966 ${ }^{7}$ und Internationales Ü̉bereinkommen zur Beseitigung jeder

\footnotetext{
${ }^{3}$ Art. 2 und 7 der Allgemeinen Erklärung der Menschenrechte, Resolution 217 A (III) vom 10.12.1948.

${ }^{4}$ Hans Werner Rengeling, Grundrechtsschutz in der Europäischen Gemeinschaft (Beck Verlag 1993) 137.

5 Oliver De Schutter, Das Diskriminierungsverbot nach dem Europäischen Menschenrechtsgesetz : seine Bedeutung für die "Rassengleichbehandlungsrichtlinie" und die Richtlinie zur Gleichbehandlung in der Beschäftigung (Amt für amtliche Veröffentlichungen der EG, 2005) 13.

${ }^{6}$ Art.14 der Allgemeinen Erklärung der Menschenrechte (n 3).

${ }^{7}$ Internationale Pakt über bürgerliche und politische Rechte vom 19.12.1966, [1973] BGBl. II 1534 .
} 
Form von Rassendiskriminierung vom 7.3.1966 zu erwähnen.

\section{EU PRIMÄRRECHT UND DIE RICHTLINIEN}

Der Schutz von Grundrechten, die auf Gleichstellung und Gleichbehandlung gerichtet sind sowie der gleichzeitige Abbau von Diskriminierungen stellen ein maßgebliches Bestreben der Europäischen Union dar. Dies spiegelt sich folglich nicht nur in den allgemeinen Rechtsgrundsätzen der Europäischen Union wider, sonder schlägt sich ebenfalls in den verschiedenen Bestimmungen der europäischen Verträge nieder. Im Besonderen zeigt sich dies in den Grundsätzen der Nichtdiskriminierung und Gleichstellung, vgl. Art. 2 des EU-Vertrages. Auch haben die Unionsmitglieder ihren Bürgern die Möglichkeit gegeben, sich auf die Menschenrechte der EMRK zu berufen (Art. 6 des EU Vertrages). An unmittelbaren unionsrechtlichen Regelungen zur Beseitigung von Diskriminierungen fehlt es hingegen lange Zeit.

So lässt sich zunächst festhalten, dass zwar einerseits EU-Maßnahmen zur Bekämpfung von Diskriminierungen und Förderung der Chancengleichheit durchaus eine europäische Tradition darstellen, sich die Gemeinschaft selbst jedoch zunächst aufgrund fehlender Ermächtigungsgrundlagen in Ihrer Rechtsetzung auf unverbindliche Erklärungen im Antidiskriminierungsbereich beschränkt hat. ${ }^{8}$

Ein Wendepunkt im Kampf mit Diskriminierungen stellte die Unterzeichnung des Vertrages von Amsterdam im Oktober 1997 dar. Mit diesem Vertrag wurden Art. 13 EGV und Art. 29 EUV als allgemeine Grundlagen zur Bekämpfung von Rassismus und Diskriminierung eingefügt. Art. 13 des Vertrages zur Gründung der Europäischen Gemeinschaft ${ }^{9}$ wurde durch Art. 19 des Vertrages über die Arbeitsweise der Europäischen Union ${ }^{10}$ ersetzt. Diese Norm enthält eine Rechtsetzungsermächtigung. Der Rat ist verpflichtet "einstimmig geeignete Vorkehrungen $z u$ treffen, um Diskriminierungen aus Gründen des Geschlechts, der Rasse, der ethnischen Herkunft, der Religion oder der Weltanschauung, einer Behinderung, des Alters oder der sexuellen Ausrichtung zu bekämpfen". ${ }^{11}$ Die Regelung des Art. 29 des Vertrages über die Europäische Union (EUV) ${ }^{12}$ (jetzt Art. 67 des Vertrages über die Arbeitsweise der Europäischen Union) nimmt Bezug auf die Verhütung und Bekämpfung von Diskriminierungen im Hinblick auf die Verwirklichung der Ziele der Union. Die im Art. 19 des Vertrages über die Arbeitsweise der Europäischen Union dargestellte Kompetenzgrundlage ermöglicht dem Rat die Regelung der Gleichbehandlungspolitik durch

\footnotetext{
${ }^{8}$ Konsolidierte Fassung des Vertrages über die Europäische Union [2002] OJ C325/1.

${ }^{9}$ Konsolidierte Fassung des Vertrages zur Gründung der Europäischen Gemeinschaft [2002] OJ C325/33.

${ }^{10}$ Konsolidierte Fassungen des Vertrags über die Europäische Union und des Vertrags über die Arbeitsweise der Europäischen Union OJ [2010] C83/47.

${ }^{11}$ Art. 19 des Vertrages über die Arbeitsweise der Europäischen Union (n 10).

${ }^{12}$ Konsolidierte Fassung des Vertrages über die Europäische Union (n 9).
} 
Rechtsakte im Sinne des Art. 288 dieses Vertrages, und auch durch andere Maßnahmen sui generis wie Aktionsprogramme oder Aktionspläne. ${ }^{13}$

Nach dem Inkrafttreten des Vertrags von Amsterdam begann die Europäische Kommission mit der Erarbeitung von Rechtsvorschriften im Bereich Antidiskriminierung. In dieser Zeit begann die Migration Policy Group zusammen mit der Europäischen Stelle für die Beobachtung von Rassismus und Fremdfeindlichkeit (EUMC) ein Forschungsprojekt, mit dem Ziel, eindeutig zu beschreiben, welcher Handlungsbedarf innerhalb der einzelnen Mitgliedstaaten besteht, um die sich entwickelnden europäischen Standards einzuhalten. ${ }^{14}$ Am 25. 09. 1999 legte die Kommission dem Rat ein umfangreiches Gesetzgebungspaket vor, das auf der Basis von Art. 13 EGV (jetzt Art. 19 Vertrag zur Gründung der Europäischen Gemeinschaft) erlassen werden sollte. Dieses Paket bestand aus:

- der Richtlinie 2000/78/EG des Rates vom 27.11.2000 zur Festlegung eines allgemeinen Rahmens für die Verwirklichung der Gleichbehandlung in Beschäftigung und Beruf ${ }^{15}$

- der Richtlinie 2000/43/EG vom 29. Juni 2000 zur Anwendung des Gleichbehandlungsgrundsatzes ohne Unterschied der Rasse oder der ethnischen Herkunft ${ }^{16}$ sowie

- einem Aktionsprogramm (zur Unterstützung und Ergänzung der Umsetzung von Richtlinien) $)^{17}$

„Wir wollen erreichen, daß überall in der Europäischen Union derselbe Schutz vor Diskriminierungen gewährleistet ist" ${ }^{\prime 18}$ - so wurde das Ziel des Maßnahmenpakets formuliert.

Die Richtlinie 2000/43/EG zur Anwendung des Gleichbehandlungsgrundsatzes ohne Unterschied der Rasse oder ethnischen Herkunft wurde am 29.06.2000 verabschiedet und umfasst die meisten Bereiche des täglichen Lebens. Der Erlaß der zweiten Richtlinie- 2000/78/EG zur Festlegung eines allgemeinen Rahmens für die Verwirklichung der Gleichbehandlung in Beschäftigung und Beruf folgte am 27.11.2000. Diese Richtlinie beschränkt sich in ihrem Regelungsumfang auf den Bereich der Beschäftigung und des Berufes. ${ }^{19}$

13 Anja Lingscheid, Antidiskriminierung im Arbeitsrecht: neue Entwicklungen im Gemeinschafts-recht auf Grund der Richtlinien 2000/43/EG und 2000/78/EG und ihre Einfügung in das deutsche Gleichbehandlungsrecht (Logos Berlin 2004) 23.

${ }^{14}$ Matthias Mahlmann, Gesetzgebung über Antidiskriminierung in den Mitgliedstaaten der EU (Jan Niessen and Isabelle Chopin, European Monitoring Centre on Racism and Xenophobia Wien 2002) 9.

${ }^{15}$ Die Richtlinie 2000/78/EG des Rates vom 27.11.2000 zur Festlegung eines allgemeinen Rahmens für die Verwirklichung der Gleichbehandlung in Beschäftigung und Beruf OJ L303/16.

16 Die Richtlinie 2000/43/EG vom 29. Juni 2000 zur Anwendung des Gleichbehandlungsgrundsatzes ohne Unterschied der Rasse oder der ethnischen Herkunft OJ L180/22.

17 Kommission, 'Ein wichtiger Schritt für die EU mehr Rechte für die Opfer von Diskriminierungen, IP/99/895,

$<$ http://europa.eu/rapid/pressReleasesAction.do?reference=IP/99/895\&format=HTML\&age $\mathrm{d}=1$ \&language $=$ DE\&guiLanguage $=\mathrm{de}>, 02.12 .2011$.

18 Die Aussage einer EU-Kommissarin Anna Diamantopolou, $<$ http://europa.eu/rapid/pressReleasesAction.do?reference=IP/99/895\&format=HTML\&age $\mathrm{d}=1$ \&language $=\mathrm{DE} \&$ guiLanguage $=\mathrm{en}>, 02.12 .2011$.

${ }^{19}$ Hannah Korthaus, Das neue Antidiskriminierungsrecht: Die Richtlinien 2000/43/EG und 2000/78/EG und die Auswirkungen auf das deutsche Arbeitsrecht (Aachen 2006) 85. 
Auf der Grundlage von Art. 141 EGV (jetzt Art. 157 des Vertrages über die Arbeitsweise der Europäischen Union) wurde die Richtlinie 2002/73/EG des Europäischen Parlaments und des Rates vom 23.09.2002 zur Änderung der Richtlinie 76/207/EWG des Rates zur Verwirklichung des Grundsatzes der Gleichbehandlung von Männern und Frauen hinsichtlich des Zugangs zur Beschäftigung, zur Berufsbildung und zum beruflichen Aufstieg sowie in Bezug auf die Arbeitsbedingungen erlassen. ${ }^{20}$ Diese wurde im Jahre 2006 durch die Richtlinie 2006/54/EG des Europäischen Parlaments und des Rates zur Verwirklichung des Grundsatzes der Chancengleichheit und Gleichbehandlung von Männern und Frauen in Arbeits- und Beschäftigungsfragen ersetzt. ${ }^{21}$ Die neue Richtlinie ersetzt eine Reihe von Richtlinien, die das Thema der Chancengleichheit von Frauen und Männer betreffen. Ziel ist die Gleichstellung der Geschlechter im Berufsleben zu gewährleisten. Daher beinhaltet die neue Regelung im wesentlichen lediglich die Neufassung bestehender Normen.

Mit gleicher Zielrichtung, nämlich die Verwirklichung des Grundsatzes der Gleichbehandlung von Männern und Frauen beim Zugang zu und bei der Versorgung mit Gütern und Dienstleistungen wurde die so genannte Gleichbehandlungsrichtlinie, die Richtlinie 2004/113/EG des Rates vom 13.12.2004 erlassen. ${ }^{22}$ Zielsetzung dieser Richtlinie ist die Bekämpfung von Diskriminierungen aufgrund des Geschlechts „,in Bereichen außerhalb des Arbeitsmarktes“. ${ }^{23}$

Allen Antidiskriminierungsrichtlinien (2000/43/EG, 2000/78/EG, 2006/54/EG, 2004/113/EG) ist ihr Ziel gemein: Die Schaffung eines Rahmens zur Bekämpfung der Diskriminierung aufgrund der Rasse, der ethnischen Herkunft, des Geschlechts, der Religion, Weltanschauung, einer Behinderung, des Alters, der Sexuellen Identität und die Einführung des Grundsatzes der Gleichbehandlung in den Mitgliedstaaten. ${ }^{24}$ Die Richtlinien unterscheiden sich hingegen hinsichtlich ihres Geltungsbereichs.

Von den unerlaubten Diskriminierungsgründen, die in den Rechtsakten genannt sind, sind die Diskriminierungsmerkmale in der Richtlinie 2000/78/EG am weitesten gefasst. Laut diesen Bestimmungen sind Diskriminierungen aufgrund der Religion, der Weltanschauung, einer Behinderung, des Alters und der sexuellen Ausrichtung verboten. Eine

\footnotetext{
${ }^{20}$ Die Richtlinie 2002/73/EG des Europäischen Parlaments und des Rates vom 23 September 2002 zur Änderung der Richtlinie 76/207/EWG des Rates zur Verwirklichung des Grundsatzes der Gleichbehandlung von Männern und Frauen hinsichtlich des Zugangs zur Beschäftigung, zur Berufsbildung und zum beruflichen Aufstieg sowie in Bezug auf die Arbeitsbedingungen [2002] OJ L269/15.

${ }^{21}$ Die Richtlinie 2006/54/EG des Europäischen Parlaments und des Rates zur Verwirklichung des Grundsatzes der Chancengleichheit und Gleichbehandlung von Männern und Frauen in Arbeits- und Beschäftigungsfragen [2006] OJ L204/23.

22 Die Richtlinie 2004/113/EG des Rates vom 13.12.2004 zur Verwirklichung des Grundsatzes der Gleichbehandlung von Männern und Frauen beim Zugang zu und bei der Versorgung mit Gütern und Dienstleistungen [2004] OJ L373/37.

23 ibid, Präambel, Punkt 9.

${ }^{24}$ Art. 1 der Gleichbehandlungsrichtlinien (n 22).
} 
weniger umfassende, aber nicht minder bedeutsame Festlegung von Diskriminierungsmerkmalen trifft die Richtlinie 2000/43. Sie verbietet Diskriminierungen aufgrund der Zugehörigkeit zu einer Rasse oder aufgrund der ethnischen Herkunft. Beide Richtlinien werden inhaltlich durch die Richtlinien 2006/54/EG und 2004/113/EG ergänzt, diese bestimmen das Geschlecht als negatives Diskriminierungsmerkmal.

Doch die Richtlinien beschränken sich nicht auf die Festlegung der Diskriminierungsmerkmale, sondern definieren unter anderem die Begriffe der mittelbaren und unmittelbaren Diskriminierung, so wird beispielsweise auch die Belästigung als eine Form von Diskriminierung eingestuft. In gleicher Weise treffen die vorgenannten Regelungen Verbote hinsichtlich der Anweisung zur Diskriminierung oder auch der Viktimisierung. Aus dem Text der Richtlinien gehen aber auch die Ausnahmen zum Nichtdiskriminierungsgrundsatz hervor, d.h. es werden Situationen gezeigt, in denen die ungleiche Behandlung gerechtfertigt sein kann.

\section{UMSETZUNG DER RICHTLINIEN IN DAS POLNISCHE RECHT}

Die Richtlinie ist ein Rechtsakt, der an die Mitgliedstaaten gerichtet ist und "hinsichtlich des zu erreichenden Ziels verbindlich" ist. Die Richtlinie wirkt nicht unmittelbar, muss aber in nationales Recht umgesetzt werden (Art. 288 des Vertrages über die Arbeitsweise der Europäischen Union). Form und Mittel der Umsetzung liegen hingegen im Ermessen der Mitgliedstaaten.

Im polnischen Recht existieren schon seit langem die Vorschriften, die Schutz vor Diskriminierungen und Gebot der Gleichbehandlung bieten. In der Verfassung, in verschiedenen Gesetzen, aber auch in von Polen ratifizierten völkerrechtlichen Verträgen, findet man die Rechtsvorschriften, die das Thema der Nichtdiskriminierung behandeln.

Gemäß Art. 32 der polnischen Verfassung: “Alle sind vor dem Gesetz gleich. Alle haben das Recht, von der öffentlichen Gewalt gleich behandelt zu werden. Niemand darf, aus welchem Grund auch immer, im politischen, gesellschaftlichen oder wirtschaftlichen Leben diskriminiert werden."25 Diese Norm beschreibt einen der wichtigsten Grundsätze - die Gleichheit aller vor dem Gesetz. Diese Norm bildet damit zugleich die Verankerung des Rechts eines jeden Einzelnen darauf, nicht diskriminiert zu werden. Die polnische Verfassung beinhaltet daneben auch Bestimmungen, die sich auf den besonderen Schutz der Interessen einiger sozialer Gruppen beziehen, beispielsweise den Schutz nationaler und ethnischer Minderheiten. Die nationalen und ethnischen Minderheiten haben das Recht auf Freiheit und Erhaltung ihrer selbst, die Entwicklung der eigenen Sprache, die Erhaltung von Bräuchen und Traditionen sowie die Entwicklung der eigenen Kultur. ${ }^{26}$ Das Verbot der Rassendiskriminierung ergibt sich hingegen nicht unmittelbar aus dem vorgenannten Rechtssatz, resultiert jedoch aus dem Verbot des Bestehens solcher Parteien, deren Programm oder Tätigkeit Rassen- und Nationalitätenhass voraussetzt oder zuläßt. ${ }^{27}$ Daneben erklärt die Verfassung

\footnotetext{
${ }^{25}$ Die Verfassung (Konstytucja RP), [1997] Dz.U. Nr 78 poz. 483.

26 ibid art 35.

27 ibid art 13 .
} 
ratifizierte Völkerrechtliche Verträge zur Quelle des allgemein geltenden Rechtes der Republik Polen (Art. 87 der Verfassung). Infolgedessen können sich die Opfer von Diskriminierungen vor dem Gericht unmittelbar auf die Verletzung der Antidiskriminierungsbestimmungen der völkerrechtlichen Verträge berufen.

Durch Änderungen des Arbeitsgesetzbuches ${ }^{28}$ wurde das polnische Arbeitsrecht an die europäischen Richtlinien angepasst. Im Januar 2004 traten die neuen Vorschriften in Kraft, die im Anschluss noch der mehrmaligen Änderung bedurften. So wurde unter anderem der Abschnitt IIa eingeführt, der die Gleichbehandlung im Rahmen von Beschäftigungsverhältnissen betrifft.

In Art. 9 § 4 des polnischen Arbeitsgesetzbuches wurde vom Gesetzgeber der Grundsatz aufgestellt, wonach die Bestimmungen der Tarifverträge und anderer auf einem Gesetz gestützter Gruppenvereinbarungen, Ordnungen und Satzungen, die Rechte und Pflichten der Parteien eines Arbeitsverhältnisses regeln, unwirksam sind, wenn sie den Grundsatz der Gleichbehandlung bei der Beschäftigung verletzen. Das allgemeine Verbot der Diskriminierung bei der Beschäftigung beinhaltet Art. $11^{3}$ des Arbeitsgesetzes. Nach dieser Vorschrift ist jede unmittelbare oder mittelbare Diskriminierung im Rahmen von Arbeitsverhältnissen auf Grundlage der unzulässigen Differenzierungsmerkmale verboten. Den Katalog der Diskriminierungskriterien wurde vom polnischen Gesetzgeber nicht im Sinne einer abschließenden Regelung gebildet, sondern erfasst die verbotenen Kriterien nur beispielhaft. So erfasst der Katalog des Gesetzgebers als maßgebliche und damit verbotene Diskriminierungsmerkmale unter anderem das Geschlecht, das Alter, eine Behinderung, die Rasse, die Religion, die Nationalität, die politische Überzeugungen, die Zugehörigkeit zu einer Gewerkschaft, die ethnische Herkunft, die Konfession, die sexuelle Orientierung und die Beschäftigung auf bestimmte oder unbestimmte Zeit oder Vollzeit- oder Teilzeitbeschäftigung. Da dies jedoch keine abschließende Enumeration darstellt, können zukünftig auch weitere Gründe für Ungleichbehandlungen ergänzt und für verboten erklärt werden. ${ }^{29}$

Art. $18^{3 \mathrm{a}}$ des Arbeitsgesetzbuches regelt den Grundsatz der Nichtdiskriminierung in der Beschäftigung. Diese Norm verbietet Diskriminierungen bei der Aufnahme und die Auflösung des Arbeitsverhältnisses, in Bezug auf Arbeitsbedingungen, Beförderung und den Zugang zu Fortbildung zum Zweck der Erhöhung der Berufsqualifikation insbesondere im Hinblick auf Geschlecht, Alter, Behinderung, Rasse, Religion, Nationalität, politische Überzeugungen, gewerkschaftliche Zugehörigkeit, ethnische Herkunft, Konfession, geschlechtliche Ausrichtung. Gemäß diesem Artikel gilt der Grundsatz der Gleichbehandlung uneingeschränkt. Folglich kommt das Gebot der Gleichbehandlung bei allen Arbeitnehmern zur Anwendung, unabhängig davon, ob jemand befristete

\footnotetext{
${ }^{28}$ Arbeitsgesetzbuch (Kodeks pracy), [1974] Dz.U. Nr 24 poz.141.

${ }^{29}$ Ludwik Florek in R Celeda, L Florek, G Goździewicz, A Hintz, A Kijowski, Ł Pisarczyk, J Skoczyński, B Wagner, T Zieliński (eds), Kodeks pracy. Komentarz (2009) 126.
} 
oder unbefristete, in Voll- oder Teilzeit beschäftigt ist. Bei Anwendung dieses Grundsatzes sind in der Regel weder die Dauer der Beschäftigung, noch eine gegebenenfalls vorhandene Berufspraxis von Bedeutung. Jedoch gilt insoweit eine sachlich gerechtfertigte Ausnahme: Da eine Beförderung von der Arbeitserfahrung des Arbeitnehmers abhängig sein kann, kann bei Beförderungsentscheidungen die vorhandene Berufspraxis berücksichtigt werden.

Der gleiche Artikel definiert daneben auch den Gleichbehandlungsgrundsatz, indem er die Begriffe der mittelbaren und unmittelbaren Diskriminierung erläutert und ausdifferenziert. Demgemäß liegt eine unmittelbare Diskriminierung dann vor, wenn ein Arbeitnehmer aus den genannten Gründen in einer vergleichbaren Situation weniger günstig behandelt wurde, wird oder werden könnte als ein anderer Arbeitnehmer. Eine mittelbare Diskriminierung liegt dagegen vor, wenn aufgrund eines scheinbar neutralen Beschlusses, angewandten Kriteriums oder einer vorgenommenen Handlung Diskrepanzen hinsichtlich der Beschäftigungsbedingungen zugunsten aller oder einer wesentlichen Zahl von Arbeitnehmern vorkommen, die zu einer aufgrund eines oder mehrerer genannten Gründe ausgesonderten Gruppe gehören, wenn diese Diskrepanzen mit anderen objektiven Gründen nicht begründet werden können. Hingegen liegt keine Diskriminierung vor, wenn die Bestimmung, das Kriterium oder die Handlung im Hinblick auf das angestrebte, rechtsmäßige Ziel objektiv begründet ist und die zur Erreichung dieses Ziels angewandten Mitteln geeignet und erforderlich sind..$^{30}$

Der polnische Gesetzgeber hat in Art. 183a $\S 5$ gleichfalls den Begriff der Belästigung und der sexuellen Belästigung am Arbeitsplatz definiert. Wird die Würde des Arbeitnehmers verletzt oder wird der Arbeitnehmer erniedrigt oder gedemütigt, so liegt darin eine Belästigung. Die Würde des Arbeitnehmers kann auf verschiedene Weise verletzt werden, z.B. durch entwürdigendes Verhalten und Bemerkungen an die Adresse des Arbeitnehmers, aber auch schon durch die Schaffung einer unangenehmen Atmosphäre am Arbeitsplatz. Gleiches gilt für Diskriminierungen wegen des Alters, des Aussehens, der Ausbildung, des Geschlechts. Unter sexueller Belästigung versteht man indes jedes inakzeptable Verhalten mit sexuellem Charakter. Dazu zählen neben sexuell bestimmten körperlichen Berührungen auch bloße Bemerkungen sexuellen Inhalts, wie beleidigende oder anzügliche Witze, Kommentare oder gar zweideutige Vorschläge. Erfasst wird insoweit nicht nur das Verhalten des Arbeitgebers, sondern auch um das Verhalten einer anderen Person, für die der Arbeitgeber verantwortlich ist. ${ }^{31}$ Die polnischen Vorschriften erweitern die Definition der Diskriminierung dadurch, dass sie auch die Anregung zur Ungleichbehandlung für eine Form der Diskriminierung halten. Das kann man für höhere Standards der Umsetzung des Gemeinschaftsrechts durch den polnischen Gesetzgeber halten.

Der Grundsatz der Gleichbehandlung im polnischen Arbeitsgesetzbuch gilt für alle Ebenen der Berufstätigkeit, für allen Stadien der Erwerbstätigkeit sowie für alle Einstellungsbedingungen. Von Letzteren werden auch das zu zahlende Entgelt, einzelne Entgeltbestandteile sowie

\footnotetext{
${ }^{30}$ Art. $18^{3 a}$ des Arbeitsgesetzbuches (n 28).

${ }^{31}$ Florek (n 29) komentarz 9.
} 
etwaige Leistungsprämien erfasst. $\mathrm{Zu}$ beachten ist ebenso, dass der Nichtdiskriminierungsgrundsatz im polnischen Arbeitsrechtrecht nicht direkt alle Tätigkeiten des Arbeitgebers im Zusammenhang mit der Begründung, Ausübung und Beendigung von Beschäftigungsverhältnissen umfasst, sondern lässt dem Katalog frei, was im Hinblick auf die europäischen Richtlinien eine großzügigere Interpretation erlaubt. So können auch die Handlungen beim Bewerbungsprozess wie z.B. Bewerbungsanzeigen Zeichen für Diskriminierung sein.

Polen hat, wie die Mehrheit der Mitgliedstaaten, Ausnahmen vom Grundsatz der Gleichbehandlung für alle in den Richtlinien genannten Merkmale zugelassen. ${ }^{32}$ So stellt Art $18^{3 \mathrm{~b}} \S 2$ des Arbeitsgesetzbuches klar, welche Handlungen nicht $\mathrm{zu}$ einer Verletzung des Grundsatzes der Gleichbehandlungen führen sollen. Danach ist eine unterschiedliche Behandlung zulässig, wenn diese durch die Art der Arbeit, die Bedingungen ihrer Verrichtung oder berufliche Anforderungen gerechtfertigt ist. Dies gilt jedoch nur, wenn der Unterscheidungsgrund eine tatsächliche und entscheidende, an den Arbeitnehmer zu stellende Berufsanforderung darstellt. So erlaubt das Gesetz beispielsweise die Kündigung der Beschäftigungsbedingungen des Arbeitnehmers hinsichtlich der Arbeitszeit aus Gründen, die die Arbeitnehmer nicht betreffen und ohne die Berufung auf die gesetzlich geregelten Diskriminierungsgründe. Gestattet ist zudem die Anwendung von Maßnahmen, die die rechtliche Situation des Arbeitnehmers aus Gründen des Schutzes der Elternschaft oder der Behinderung notwendigerweise differenzieren. Gleiches gilt für die unterschiedliche Behandlung der Arbeitnehmer aus Gründen des Alters, deren Zweck die Erhöhung der Berufsqualifikation ist. Dem Arbeitgeber bietet sich die Möglichkeit zwischen den Arbeitnehmern bezüglich ihrer Eigenschaften und Qualifikationen zu differenzieren, soweit diese an bestimmten Arbeitsplätzen erforderlich sind - z.B. eine entsprechende Ausbildung, Fremdsprachenkenntnisse usw. Berufliche Anforderungen können auch Charaktereigenschaften sein, wie z. B. Höflichkeit, Freundlichkeit, Kommunikationsfähigkeit usw. Die Differenzierung der Arbeitnehmer kann nur aufgrund der objektiven Kriterien durchgeführt werden. Dabei ist zu berücksichtigen, dass die Beweislast auf der Seite des Arbeitgebers liegt, da er beweisen muss, dass er objektiv gehandelt hat.

Ebenfalls von großer praktischer Bedeutung sind die Regelungen des polnischen Rechts, die eine Ungleichbehandlung aufgrund konfessioneller bzw. religiöser Einstellungen und Tendenzen innerhalb von Beschäftigungsverhältnissen oder deren Begründung gestatten, sofern einer der Vertragspartner eine Kirche oder ein Konfessionsbund ist. Ausnahmsweise darf hier aufgrund der Religion oder Konfessionszugehörigkeit des Arbeitnehmers eine Ungleichbehandlung erfolgen, stets jedoch unter der Voraussetzung, dass die Religion oder Konfession des Arbeitnehmers ein wesentliches, begründetes und

\footnotetext{
32 Janet Cormack, Mark Bell, Entwicklung des Antidiskriminierungsrecht in Europa (Migration Policy Group 2005) 55.
} 
berechtigtes Berufsanforderung darstellt. ${ }^{33}$ Hier stellt sich die Frage, ob z.B. Nichtbeschäftigung von Pädagogen in Religionsgemeinschaften, die einer anderen Glaubensgemeinschaft angehören gerechtfertigt ist. Man soll annehmen, dass es in den Regelungen um die Stellen geht, die direkt mit der Religion oder Konfession verbunden sind.

Der polnische Gesetzgeber erlaubt bezüglich der europarechtlichen Normen die Einführung der Vorschriften in die nationale Rechtsordnung, die die Durchführung der sog. positiven Maßnahmen ermöglichen, mit dem Zweck, die Chancen für alle oder für eine wesentliche Anzahl von Arbeitnehmern auszugleichen und schafft damit eine - zumindest vorübergehende Möglichkeit zum Ausgleich bestehender Nachteile, ähnlich der positiv ausgerichteten Maßnahmen im Sinne des $\S 5$ AGG im deutschen Recht. Im Gegensatz zur dortigen Regelung können die Maßnahmen im Geltungsbereich des polnischen Rechts jedoch nur für eine bestimmte Zeit getroffen werden. Beiden ist jedoch gemein, dass sie den Zweck verfolgen sollen die Chancen und bestehenden Nachteile aller oder einer großen Zahl von Arbeitnehmern auszugleichen (Art. $18^{3 \mathrm{~b}} \S 3$ ). Für das polnische Recht gilt insoweit, dass die Arbeitnehmer im Hinblick auf Alter, Mutterschaftsschutz, Behinderung günstiger behandelt werden. In praktischer Hinsicht entfaltet die Möglichkeit mittels positiver oder spezieller Maßnahmen bestehende Benachteiligungen für behinderte Menschen auszugleichen die größte Relevanz und den häufigsten Anwendungsfall. ${ }^{34}$ Der Staat garantiert den behinderten Menschen und auch den Arbeitgebern, die jene beschäftigen, bestimmte Sonderrechte, die die Integration der Behinderten am Arbeitsplatz erleichtern sollen. Die Behandlung der Menschen mit Behinderungen wurde im Gesetz über berufliche und soziale Rehabilitation und die Beschäftigung von Menschen mit Behinderungen $^{35}$ geregelt. Das Gesetz - zusammen mit zahlreichen Durchführungsakten - das darf insoweit festgehalten werden, erfüllt die Anforderungen der beiden Gleichbehandlungsrichtlinien, bezugnehmend auf Nichtdiskriminierung wegen der Behinderung. Diese Normen umfassen die Regelungen, die z.B. Zugang zu Gütern und Dienstleistungen, öffentlichen Gebäuden, Anpassung der Arbeitsplätze betreffen. Die Hauptinstitutionen von sozialer und beruflicher Integration der Behinderten sind die geschützten Betriebe. Diese Betriebe erhalten Zuschüsse und Steuervergünstigungen gemäß der Zahl der eingestellten Behinderten, so dass der polnische Staat nicht nur der rechtliche Rahmen festgelegt hat, sondern auch zahlreiche Möglichkeiten zur faktischen Beseitigung der bestehenden Nachteile geschaffen hat.

Zahlreiche positive Maßnahmen wurden auch für den von Nachteilen betroffenen Bereich der ethnischen Minderheiten geschaffen, im Besonderen für die Bevölkerungsgruppe der Roma. So kündigte die polnische Regierung im Jahr 2004 unter anderem ein Programm für die Roma-Gemeinschaft an, dass die Roma in folgenden Bereichen unterstützen soll: Bildung, Gesundheitsführsorge, Lebensbedingungen, Kultur, Bekämpfung der

\footnotetext{
${ }^{33}$ Art $18^{3 \mathrm{~b}} \S 4$ des Arbeitsgesetzbuches (n 28).

${ }^{34}$ Indra Burg, Positive Maßnahmen zwischen Unternehmerfreiheit und Gleichbehandlung, (Duncker \& Humblot Berlin 2009) 50.

${ }^{35}$ Ustawa z dnia 27 sierpnia 1997 r. o rehabilitacji zawodowej i społecznej oraz zatrudnianiu osób niepełnosprawnych, [1997] Dz.U. Nr 123 poz. 776 .
} 
Arbeitslosigkeit. ${ }^{36}$ Die Fortsetzung dieses Programmes von Jahr zu Jahr hat zu der Verbesserung der Situation von Romagemeinschaft einen sehr grossen Beitrag geleistet. Infolgedessen wurden neue Arbeitsplätze für Roma geschafft, Zugang zu dem Gesundheitswesen wurde erleichtert, auch Bildungsmöglichkeiten wurden verbessert. ${ }^{37}$

Die polnischen arbeitsrechtlichen Vorschriften regulieren auch den Schadenersatzanspruch der Arbeitnehmer, die innerhalb ihres Beschäftigungsverhältnisses diskriminiert wurden. Gemäß Art.183d hat der Arbeitnehmer einen Anspruch auf Schadensersatz, in einer Höhe, die nicht niedriger sein darf als der in gesonderten Vorschriften bestimmtem Mindestlohn (Monatslohn). ${ }^{38}$

Es ist an dieser Stelle zu erwähnen, das sich das polnische Arbeitsgesetzbuch nur mit der Gleichbehandlung in Bezug auf Arbeitsverhältnisse befasst. D.h. Die Antidiskriminierungsvorschriften des Arbeitsgesetzbuches gelten nicht für andere Verhältnisse wie z.B. Dienstvertrag, Werkvertrag u.s.w.

Es gibt ferner mehrere Vorschriften in anderen Gesetzen, die sich mit der Thematik der Nichtdiskriminierung mehr oder weniger befassen. Hier unterscheidet man z.B.: das Gesetz vom 20. 04. 2004 über Beschäftigungsförderung und die Arbeitsmarktinstitutionen (enthält ein allgemeines Diskriminierungsverbot) ${ }^{39}$, das Gesetz über nationale und ethnische Minderheiten und über Regionalsprachen (in Kraft seit 1. 05. 2005). ${ }^{40}$ Am 03.12.2010 wurde das Gesetz über die Umsetzung von einigen EU-Vorschriften in dem Bereich der Gleichbehandlung erlassen.

\section{Deutsches AGG. Die UMSetzung DeR ANTIDISKRIMINIERUNGSRICHTLINIEN IN DAS DEUTSCHE RECHT}

Im Gegensatz zu Polen wurden in Deutschland die Nichtdiskriminierungsbestimmungen in einem Gesetz konzentriert. Das Allgemeine Gleichbehandlungsgesetz $(\mathrm{AGG})^{41}$ ist am 18. August 2006 in Kraft getreten und betrifft nicht nur Beschäftigungsbereich sondern auch andere Bereiche wie beispielsweise den Zugang zur Versorgung mit Gütern und Dienstleistungen im Rahmen der öffentlichen Daseinsvorsorge. Das Gesetz lässt sich in vier Teile unterteilen: Allgemeiner Teil (§1-5), Arbeitsrechtlicher Teil (§6-8), Zivilrechtlicher Teil (§§19-21), ein Teil über

\footnotetext{
${ }^{36}$ Cormack, Bell (n 32) 71.

${ }^{37}$ www.msw.gov.pl

${ }^{38}$ Art. $18^{3 \mathrm{~d}}$ des Arbeitsgesetzbuches (n 28).

${ }^{39}$ Ustawa z dnia 20 kwietnia 2004 o promocji zatrudnienia i instytucjach rynku pracy, [2004] Dz.U. Nr 99 poz. 1001.

${ }^{40}$ Ustawa o mniejszościach narodowych i etnicznych oraz o języku regionalnym, [2005] Dz.U. Nr 17, poz. 141 i Nr 62, poz. 550.

${ }^{41}$ Gesetz zur Umsetzung europäischer Richtlinien zur Verwirklichung des Grundsatzes der Gleichbehandlung, [2006] BGBl. I 1897.
} 
Rechtsschutz (§§ 22 und 23), Sonderregelungen und Regelungen über Antidiskriminierungsstelle.

Daneben gibt es im deutschen Rechtssystem natürlich auch weitere Vorschriften, die Schutz vor Diskriminierungen gewährleisten und die in anderen Gesetzen zu finden sind. An dieser Stelle soll man vor allem Art. 3 des Grundgesetzes für die Bundesrepublik Deutschland nennen, der sowohl die Gleichheit vor dem Gesetz garantiert, als auch Benachteiligungen aufgrund von Geschlecht, Abstammung, Rasse, Glauben etc. untersagt. ${ }^{42}$

Das Ziel des Allgemeinen Gleichbehandlungsgesetzes ist es zunächst Benachteiligungen wegen der im Gesetz bestimmten Gründe zu verhindern oder zu beseitigen. Die unerlaubten Diskriminierungsmerkmale gemäß $\S 1$ AGG sind: die Rasse, die ethnische Herkunft, das Geschlecht, die Religion, die Weltanschauung, die Behinderung, das Alter und die sexuelle Identität. Der Anwendungsbereich des Gesetzes ist breit und bezieht sich unter Anderem auf: Zugang $\mathrm{zu}$ unselbstständiger und selbstständiger Erwerbstätigkeit, die Beschäftigungs- und Arbeitsbedingungen, Zugang zu der Berufsberatung und Berufsbildung, die Mitgliedschaft und Mitwirkung in einer Beschäftigten- oder Arbeitgebervereinigung, den Sozialschutz, die sozialen Vergünstigungen, die Bildung, öffentlichen Gütern und Dienstleistungen ( $§ 2$ AGG). Dies umfasst auch privatrechtlich $\mathrm{zu}$ beurteilende Schuldverhältnisse. Eine bedeutsame Ausnahme vom Anwendungsbereich des AGG ist jedoch in $\S 2$ Abs. 4 AGG geregelt. Dieser lautet: „Für Kündigungen gelten ausschließlich die Bestimmungen zum allgemeinen und besonderen Kündigungsschutz." Das bedeutet, dass man für Kündigungen nicht AGG sondern Kündigungsschutzgesetz anwenden soll.

Das Gesetz beinhaltet eine komplexe Regelung, ist aber - wie oben erwähnt wurde - keine abschließende Regelung des Schutzes vor Benachteiligung innerhalb des deutschen Rechtssystems. Die in anderen Rechtsvorschriften existierenden Benachteiligungsverbote und Gleichbehandlungsgebote gelten neben dem AGG ( $\$ 2$ Abs. 3 AGG). ${ }^{43}$

$\mathrm{Zu}$ beachten sind die Abweichungen von der Begrifflichkeit der Diskriminierung in dem AGG gegenüber der Begriffsbestimmung der europäischen Richtlinien. Das deutsche AGG verwendet, anders als die Richtlinien, den Begriff der Benachteiligung. Der deutsche Gesetzgeber wollte dadurch verdeutlichen, dass nicht jede unterschiedliche Behandlung, die für den Betroffenen mit einem Nachteil verbunden ist, auch eine Diskriminierung ist. $^{44}$ Die Benachteiligung wird nach dieser Betrachtungsweise erst zur Diskriminierung wenn sie nicht gerechtfertigt sein könnte. ${ }^{45}$

Der erste Teil des Gesetzes - Allgemeiner Teil - beinhaltet ähnlich wie die polnische Vorschriften die Definitionen von vier Formen von Benachteiligung: unmittelbare Benachteiligung, mittelbare Benachteiligung, Belästigung und sexuelle Belästigung. Als Benachteiligung gilt - ebenso wie im polnischen Recht - auch die Anweisung zur Benachteiligung einer Person

\footnotetext{
${ }^{42}$ Grundgesetz für die Bundesrepublik Deutschland, [2006] BGBl. I 2034.

${ }^{43}$ Christian Oberwetter, Allgemeines Gleichbehandlungsgesetz (AGG) Kommentar (Stand: 1. November 2006) (R. S. Schulz GmbH 2006) 10.

${ }^{44}$ Max Mälzer, Der Tatbestand der Benachteiligung nach dem AGG (Grinedn, 2008) 1.

45 Klaus Hümmerich, Winfried Boecken, Franz Josef Düwell, AnwaltKommentar Arbeitsrecht (Deutscher Anwaltverlag 2007) 52.
} 
(§ 3 AGG). Diese Begriffe wurden aus den europäischen Richtlinien übernommen (vergleichbare Begriffsbezeichnung befindet sich in dem polnischen Arbeitsgesetz auch aus den Richtlinien) und gelten nicht nur für den Bereich der Beschäftigung sondern für alle andere Bereiche, die das Gesetz erfasst.

Die direkteste Form der Benachteiligung ist eine unmittelbare Benachteiligung. Diese liegt vor, wenn eine Person wegen eines in dem Gesetz genannten Grundes eine weniger günstige Behandlung erfährt, als eine andere Person in einer vergleichbaren Situation erfährt, erfahren hat oder erfahren würde. Das betrifft auch die ungünstigere Behandlung einer Frau wegen Schwangerschaft oder Mutterschaft. Praktische Anwendung des Verbots der unmittelbaren Diskriminierung zeigt sich bei einer zweistufigen Prüfung: Zunächst ist die Kausalität zu prüfen also die weniger günstige Behandlung wegen eines Diskriminierungsmerkmals. Wird ein solches Anknüpfen an ein Diskriminierungsmerkmal des $\S 1$ AGG bejaht, ist die Kausalität des Anknüpfens an ein solches Merkmal für die getroffenen Maßnahme zu prüfen. Die Kausalität wird wiederum durch den im Vergleich mit einer konkreten (gegenwärtigen oder vergangenen) oder hypothetischen Vergleichsperson ermittelt. Letztlich bleibt $\mathrm{zu}$ prüfen, ob eine solche unmittelbare Diskriminierung ausnahmsweise gerechtfertigt werden kann. Wenn eine Benachteiligung mittels unterschiedlicher Behandlung vorliegt, soll zunächst untersucht werden, ob eine konkrete Vergleichsperson günstiger behandelt wird. Eine unmittelbare Diskriminierung liegt dann vor wenn sich diese konkrete Vergleichsperson aufgrund der Bezugnahme auf das Diskriminierungsmerkmal von der benachteiligten Person unterscheidet und in sonstiger Hinsicht vergleichbar ist. ${ }^{46}$

Eine mittelbare Diskriminierung ist dann, wenn „dem Anschein nach neutrale Vorschriften, Kriterien oder Verfahren” Personen wegen des in dem Gesetz genannten Grundes ,gegenüber anderen Personen in besonderer Weise benachteiligen können" ( $(3$ Abs. 2 AGG). Das unzulässige Differenzierungsmerkmal bildet hier nicht das Entscheidungskriterium, weil sich die Diskriminierung erst als faktische Folge ergibt. Wichtig ist, ob sich eine scheinbar neutrale Regelung oder Verhaltensweise so auswirkt, dass die Personen, die ein Diskriminierungsmerkmal erfüllen ungünstiger behandelt werden können. ${ }^{47}$ Das gilt aber nicht für die Situation wenn die betreffenden Vorschriften, Kriterien oder Verfahren durch ein rechtmäßiges Ziel sachlich gerechtfertigt sind und die angewandte Mittel zur Erreichung dieses Ziels

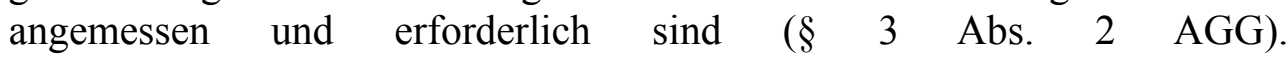
Tatbestandsvoraussetzung ist also hier das Fehlen von Rechtfertigungsgründen. ${ }^{48}$ Als Beispiel für eine mittelbare Diskriminierung z.B. aufgrund des Geschlechts gilt eine Benachteiligung von Teilzeitbeschäftigten, wenn es sich bei Teilzeitbeschäftigten überwiegend um

\footnotetext{
${ }^{46}$ Dagmar Schiek, Eva Kocher, Silke Laskowski, Felix Welti, Marlene Schmidt, AGG Ein Kommentar aus Europäischer Perspektive (Sellier European Law Publishers 2007) 115.

47 Oberwetter (n 43) 12.

${ }^{48}$ Hümmerich, Boecken, Düwell (n 45) 61.
} 
Frauen handelt. ${ }^{49}$ Ein weiteres Beispiel - in der Stellenausschreibung für einen Posten soll eine langjährige, ununterbrochene Vollzeittätigkeit in dem bestimmten Bereich nachgewiesen werden. Da die Frauen oft haben im Zusammenhang mit der Geburt von Kindern wesentlich häufiger Phasen von Teilzeitbeschäftigung oder einfach Lücken in der Beschäftigung haben kann in diesem Fall eine mittelbare Benachteiligung von Frauen vorliegen. ${ }^{50}$

Eine Belästigung liegt nach Maßgabe des deutsches Gesetzes ( $§ 3$ Abs. 3 AGG) dann vor, wenn unerwünschte Verhaltensweisen, die mit den unzulässigen Differenzierungskriterien verbunden sind, bezwecken oder bewirken, dass die Würde der betreffenden Person verletzt wird und ein von Einschüchterungen, Anfeindungen, Erniedrigungen, Entwürdigungen oder Beleidigungen gekennzeichnetes Umfeld geschaffen wird. Ähnlich ist sexuelle Belästigung definiert, das unerwünschte Verhalten ist aber sexuell bestimmt ( $\$ 3$ Abs. 4 AGG). Die Belästigung im Sinne des Gesetzes ist also mit dem Angriff auf die Persönlichkeit verbunden. Hier, anders als bei den unmittelbaren und mittelbaren Diskriminierungen, müssen keine Vergleichsgruppen gebildet werden. ${ }^{51}$ Wesentlich ist die Verletzung der Würde einer Person durch unerwünschte Verhaltensweisen wie z.B. Einschüchterungen, Anfeindungen, Erniedrigungen, Beleidigungen. ${ }^{52}$

Laut dem deutschen Gesetz ist auch- wie oben schon erwähnt wurdedie Anweisung zur Benachteiligung einer Person eine Form der Diskriminierung. Die Anweisung muss aber mit Vorsatz erfolgen, d.h. der Anweisende muss wissen und wollen, dass der Angewiesene die Handlung ausführt. ${ }^{53}$

Bei einer Analyse der deutschen Vorschriften zeigt sich der Begriff einer Mehrfachdiskriminierung. Während die Richtlinien keine besonderen Regelungen in diesem Bereich beinhalten, hat der deutsche Gesetzgeber diese Problematik in das AGG aufgenommen (§ 4 des Gesetzes regelt unterschiedliche Behandlung wegen mehrerer Gründe bzw. Diskriminierungsmerkmale). Diese Form von (Mehrfach-)Diskriminierung tritt beispielsweise dann auf, wenn bestimmte Personen oder Personengruppen gleichzeitig aus mehreren Gründen benachteiligt werden z.B. behinderte Frauen können sowohl aufgrund des Geschlechts als auch aufgrund der Behinderung ungleich behandelt werden. Die erforderliche Rechtfertigung der Ungleichbehandlung liegt jedoch nur dann vor, wenn sich die sachliche Rechtfertigung auf alle Gründe und nicht nur auf einen Einzelnen erstreckt. Der polnische Gesetzgeber hat hingegen auf eine ähnliche Regelungen verzichtet - die Mehrfachdiskriminierung ist in dem polnischen Arbeitsgesetzbuch nicht geregelt.

Das deutsche Gesetz regelt eine Reihe von Rechtfertigungsgründen für Benachteiligungen, die in den $\S \S 8 \mathrm{ff}$. AGG genannt sind. Eine unterschiedliche Behandlung ist gemäß $\S 8$ AGG zulässig, wenn sie wegen der Art der auszuübenden Tätigkeit oder der Bedingungen ihrer Ausübung

\footnotetext{
${ }^{49}$ Eva Hohmann, Das allgemeine Gleichbehandlungsgesetz (agg) - Handlungsanweisungen für die Praxis (GRIN Verlag 2008) 39.

${ }^{50}$ Holger Schwarz, Diskriminierungsfreie Personalrekrutierung im Rahmen des § 11 AGG unter gleichzeitiger Betrachtung haftungsrechtlicher Fragen des BGB (GRIN Verlag 2008) 24.

${ }^{51}$ Hohmann (n 49) 12.

52 ibid 39.

53 ibid.
} 
eine wesentliche und entscheidende berufliche Anforderung darstellt, sofern der Zweck rechtmäßig und die Anforderung angemessen sind. Zum Beispiel ist eine unterschiedliche Behandlung dann zulässig wenn ein Model für Damenbekleidung gesucht wird und somit das Geschlecht eine wesentliche und entscheidende berufliche Anforderung darstellt. Gleiches gilt, wenn für eine männliche Rolle ein männlicher Darsteller gesucht wird. Es ist daher darauf achten, dass die Angemessenheit immer bezogen auf den Einzelfall geprüft werden im Streitfall letztlich auch einer arbeitsgerichtlichen Nachprüfung standhalten muss. ${ }^{54}$ In der Literatur findet sich oftmals das folgende Beispiel: In einem chinesischen Restaurant werden Kellner gesucht, der Arbeitgeber fordert in einer Stellenausschreibung nur Asiaten zur Bewerbung auf. In diesem Fall stellt sich die Frage, ob eine unterschiedliche Behandlung (aufgrund der ethnischen Herkunft) begründet ist. Dies ist umstritten. Allein das Betreiben eines Restaurants mit ausländischen Speisen führt wohl nicht $\mathrm{zu}$ einer Rechtfertigung der Differenzierung. ${ }^{55}$ Die Differenzierung könnte jedoch im genannten Beispiel gleichwohl gerechtfertigt sein, sofern sich der Arbeitgeber auf die Erwartungen der Kunden berufen kann, die ein chinesische Restaurant wegen der authentischen Atmosphäre besuchen.

Weitere Abweichungen von dem Diskriminierungsverbot sind in den $\S 9$ und $\S 10$ des AGG geregelt. Die Regelung des $\S 9$ AGG richtet sich an Religionsgemeinschaften. Ähnlich wie in den Richtlinien, ist eine unterschiedliche Behandlung wegen der Religion oder der Weltanschauung bei der Beschäftigung durch Religionsgemeinschaften zulässig, wenn eine bestimmte Religion oder Weltanschauung unter Beachtung des Selbstverständnisses der jeweiligen Religionsgemeinschaft oder Vereinigung im Hinblick auf ihr Selbstbestimmungsrecht oder nach der Art der Tätigkeit eine gerechtfertigte berufliche Anforderung darstellt.

Der Regelungsinhalt des $\S 10$ AGG richtet sich auf die zulässige Ungleichbehandlung wegen des Alters des Betroffenen. Eine Ungleichbehandlung wegen des Alters ist nur dann zulässig, wenn sie objektiv, angemessen und durch ein legitimes Ziel gerechtfertigt ist. Diese Vorschrift regelt auch Beispiele für gerechtfertigte, unterschiedliche Behandlungen. Z.B. ist Laut diesem Vorschrift die Festsetzung eines Höchstalters für die Einstellung aufgrund der spezifischen Ausbildungsanforderungen eines bestimmten Arbeitsplatzes oder aufgrund der Notwendigkeit einer angemessenen Beschäftigungszeit vor dem Eintritt in den Ruhestand zulässig.

Das Gesetz regelt auch Organisationspflichten des Arbeitgebers wie z.B die Beachtung von Benachteiligungsverbote bei Stellenausschreibungen (§ 11 AGG), die Verpflichtung des Arbeitgebers erforderlichen Maßnahmen zum Schutz vor Benachteiligungen zu treffen ( $\$ 12$ AGG), Rechte der Beschäftigten (Beschwerderecht $\S 13$ AGG, Leistungsverweigerungsrecht $\S$

\footnotetext{
${ }^{54}$ ibid 7.

${ }^{55}$ Schwarz (n 50) 46.
} 
14 AGG), Schadensersatz- und Entschädigungsansprüchen (§ 15 AGG).

Das Gebot der Nichtdiskriminierung des Allgemeinen Gleichbehandlungsgesetzes sind, wie bereits erwähnt, nicht nur auf den Regelungsbereich der Arbeitsverhältnisse beschränkt, sondern gelten auch in den übrigen Bereichen des Zivilrechts, vgl. §§ 19-21 AGG. Für diese Regelungen ist jedoch zu konstatieren, dass sie über die europarechtlichen Vorgaben hinausgehen. In dem polnischen Arbeitsgesetzbuch findet man solche Regelungen nicht. Das Arbeitsgesetzbuch (und auch die Diskriminierungsverbote) gilt nur für Arbeitsverhältnisse, also die Verhältnisse, die aufgrund des Arbeitsvertrages zustande gekommen sind. Infolgedessen sind die Personen, die z.B. im Rahmen eines Dienstvertrages tätig sind, nicht durch die arbeitsrechtliche Antidiskriminierungsvorschriften geschützt.

\section{ZUSAMMENFASSUNG}

Eine Analyse von oben erwähnten Rechtsakten zeigt, dass sowohl Deutschland als auch Polen rechtlichen Schutz vor Diskriminierung bieten. Deutschland hat diese Problematik in das neue Gesetz - das AGG aufgenommen. Polen hat die europäischen Richtlinien hingegen durch die Änderung bereits bestehender Vorschriften des Arbeitsgesetzbuches umgesetzt. Das in Polen neu erlassene Gesetz (im Jahre 2010) über die Umsetzung von einigen europäischen Vorschriften betrifft nicht das Arbeitsleben. Auf jeden Fall werden die gesetzlichen Regelungen zum Diskriminierungsschutz in dem Bereich Arbeitsrecht sowohl in Polen als auch in Deutschland komplex aufgefasst. Die Definitionen verschiedener Formen der Diskriminierung, aber auch die Ausnahmen von Diskriminierungsverboten sind in beiden Rechtssystemen aus den Richtlinien übernommen und daher ähnlich geregelt. Es gibt aber auch Besonderheiten, die anders gefasst wurden, wie z.B. der Begriff der Mehrfachdiskriminierung in dem deutschen Gesetz oder der offene Katalog von unzulässigen Differenzierungskriterien in dem polnischen Arbeitsrecht. Ferner bedarf es eines kurzen Kommentars bezüglich der Umsetzungsweise der europäischen Richtlinien. Die Umsetzung der Antidiskriminierungsrichtlinien im deutschen Recht mittels eines einheitlichen Gesetzes ist positiv hervorzuheben. Da das europäische Diskriminierungsverbot nicht nur streng an Arbeitsverhältnisse anknüpft, sondern auch andere zivilrechtliche Bereiche erfassen soll, sind die Regelungen eindeutiger und damit einer stringenteren sowie vereinfachten praktischen Anwendung zuzuführen, sobald sie in einem Gesetz gesammelt sind. Im polnischen Recht muss man in anderen Rechtsakten nach entsprechenden Normen suchen, wenn die Ungleichbehandlung nicht direkt das Arbeitsverhältnis betrifft. Dies erschwert die praktische Umsetzung und damit auch Anwendung der entsprechenden Regelungen.

Bei näherer Betrachtung der Anwendung der Antidiskriminierungsvorschriften stellt sich letztlich die Frage der Praxistauglichkeit, nämlich, ob die Regelungen - ob im polnischen oder deutschen Recht - dem Betroffenen tatsächlich einen umfassenden Schutz vermitteln können. Die Antwort ist auf jeden Fall nicht einfach. 
In den letzten Jahren lässt sich eine positive Entwicklung erkennen wenn es um Kampf gegen Diskriminierungen in den Mitgliedstaaten geht. Sowohl auf nationaler als auch auf europäischer Ebene werden neben dem Erlass entsprechender rechtlicher Rahmen auch zahlreiche verschiedene flankierende Programme und Kampagnen durchgeführt, die das Ziel haben, das Thema der Diskriminierung und deren Bekämpfung den Menschen näher zu bringen. Hier ist z.B. Informationskampagne „Für Vielfalt. Gegen Diskriminierung.“ zu erwähnen.

Alle Tätigkeiten und Bestrebungen, ob rechtlicher oder tatsächlicher Natur, in diesem Bereich spielen eine zunehmend wichtige Rolle. Im Ergebnis bleibt daher festzuhalten werden, dass der bestehende rechtliche Rahmen zur Bekämpfung von Diskriminierungen innerhalb der europäischen Mitgliedsstaaten, insbesondere aber in Polen und Deutschland, den Anforderungen der Moderne genügt, dennoch ein Handlungs- und Informationsbedarf auf Seiten der Bürger besteht, denn nur wenn Menschen ihre Rechte kennen, können sie diese auch wahrnehmen. 